\title{
Complex inorganic structures from powder diffraction: case of tetrahydroborates of light metals
}

\author{
Radovan Černý ${ }^{*, I}$ and Yaroslav Filinchuk, II \\ I Laboratory of Crystallography, University of Geneva, 1211 Geneva, Switzerland

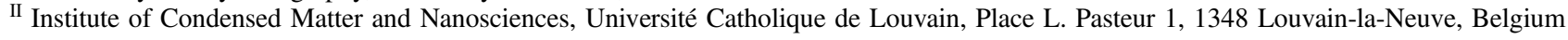

Received March 31, 2011; accepted September 29, 2011

\section{Powder diffraction / Structure solution / Hydride / Rietveld refinement}

\begin{abstract}
Powder diffraction plays a central role in the characterization of light metal tetrahydroborates (borohydrides), novel boron based hydrides recognized as a potential solution for hydrogen storage. Numerous novel $\mathrm{BH}_{4}^{-}$ based materials have been investigated during the past few years and this class of materials has a fascinating structural chemistry. We review the powder diffraction methods, problems and solutions which are specific for these weakly diffracting and badly crystallized materials. Examples of highlights and pitfalls of the powder diffraction are given. Complex structures with as many as 55 independent atoms were fully characterized, and $P-T$ phase diagrams accessible from in-situ powder diffraction enable understanding of these ionic crystals with important directional bonding.
\end{abstract}

\section{Introduction}

Tetrahydroborates are largely ionic compounds with a general formula $M\left(\mathrm{BH}_{4}\right)_{n}$, consisting of metal cations $M^{n+}$ and borohydride anions $\mathrm{BH}_{4}^{-}$. Mono- and double-cation tetrahydroborates are known. Tetrahydroborates are commonly called borohydrides, and we will use this name in this review even if it does not follow the IUPAC terminology (Adams, 1972). Light metal borohydrides (where $M$ is $2^{\text {nd }}-4^{\text {th }}$ period metal) are used as reducing agents, starting compounds for the synthesis of organometallic derivatives, precursors for the production of borides, hydrides, as well as other inorganic materials, and catalysts for hydrogenation, isomerisation, oligomerisation, and polymerization. Due to a high weight percent of hydrogen, they are considered as prospective hydrogen storage materials. Indeed, some borohydrides desorb a large quantity of hydrogen (up to $20.8 \mathrm{wt} \%$ ), although the decomposition temperatures are usually high. Many light metal and transition metal borohydrides are known from the literature, for reviews see (James and Wallbridge, 1970; Marks and Kolb,

* Correspondence authors (e-mail: Radovan.Cerny@unige.ch, Yaroslav.Filinchuk@uclouvain.be)
1977; Grochala and Edwards, 2004; Filinchuk et al., 2010a; Ravnsbæk et al., 2010a). Almost all borohydrides are crystalline solids already at room temperature. This gives an advantage of using diffraction methods to study their crystal structure. However, only very few structural characterizations had been done until recently. The number of solved structures increased dramatically with with the introduction of modern powder diffraction methods, as it has been done for the first time for $\mathrm{LiBH}_{4}$ (Soulié et al., 2002).

We will not review here the preparation of borohydrides. For a recent review on synthetic approaches to inorganic borohydrides see (Hagemann and Černý, 2010). During the past decades mechanochemistry, such as ball milling has become increasingly important, and the majority of the novel metal borohydrides are synthesized by this solvent-free method (Suryanarayana, 2001, Balema et al., 2002). As the synthesis products are homogeneous and fine, but contain multiple and not always well crystallized phases, their structural characterization becomes a challenge for $a b$ initio powder diffraction methods. Here we review the application of these methods to borohydrides, and give the guidelines for a successful structure solution and refinement.

\section{Localization of hydrogen atoms from X-ray data}

In the case of lightweight hydrides, hydrogen becomes a non-negligible X-ray scatterer, i.e. for $\mathrm{LiBH}_{4}$, the lightest possible borohydride, hydrogen atoms have been located unambiguously by PXD alone (Soulié et al., 2002). The contribution of hydrogen to the intensity scattered by $\mathrm{LiBH}_{4}$ is $26 \%$ for $\mathrm{X}$-rays compared to $52 \%$ for neutrons (Filinchuk et al., 2008a). Although the precision of the metal-hydrogen bond lengths obtained from PXD data are lower than that usually attained by neutron diffraction. The true positions of $\mathrm{H}$-atoms determined from X-ray diffraction data are displaced from $\mathrm{B}$-atom along the $\mathrm{B}-\mathrm{H}$ bond by $\sim 0.08 \AA$ due to the displacement of the electron cloud, and by $\sim 0.034-0.10 \AA$ due to the libration motion of $\mathrm{BH}_{4}$ groups (Fig. 1), which is both temperature- and structure-dependent (Filinchuk et al., 2008a; Filinchuk et al., 2010a). Recently even charge densities in $\mathrm{LiBD}_{4}$ 

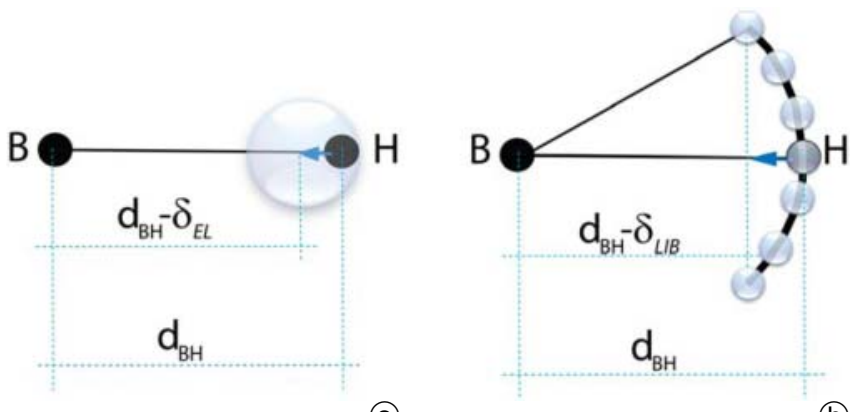

(a)

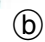

Fig. 1. Schematic illustration of systematic differences between $B-H$ distances determined by various methods. A discrepancy $\delta_{\mathrm{EL}}$ between the $\mathrm{B}-\mathrm{H}$ bond lengths determined from X-ray and neutron diffraction (a) comes from the displacement of the electron cloud (observed by X-ray diffraction) relative to the average nuclear position (seen by diffraction of neutrons). This difference is temperature independent, $\delta_{\mathrm{EL}}=0.08 \AA$ (Filinchuk et al., 2008a). A discrepancy $\delta_{\mathrm{LIB}}$ between DFT calculations and the experimentally determined $\mathrm{B}-\mathrm{H}$ bond lengths originates also from the geometric effect $(\mathbf{b})$ caused by a libration of the $\mathrm{BH}_{4}$ unit. $\delta_{\mathrm{LIB}}$ is temperature dependent, specific to each crystal structure, and dependent on the mass of the H-isotope. Note that for the X-ray diffraction data both corrections have to be applied.

have been reliably extracted from the synchrotron powder diffraction data with the high counting statistics by using the MEM analysis (Buchter et al., 2011).

Hydrogen desorption from stoichiometric hydrides is typically associated with significant changes in both composition and structure, very often involving well crystalline phases. Therefore, even though hydrogen has the lowest X-ray scattering power of any element, one can clearly observe hydrogen absorption and desorption as structural changes (Hauback, 2008; Gray et al., 2006; Orimo et al., 2007).

The acquisition of neutron powder diffraction data from borohydrides is complicated by the need for isotopically labeled samples (containing ${ }^{11} \mathrm{~B}$ and ${ }^{2} \mathrm{H}$ ) to avoid the high neutron absorption or incoherent neutron scattering from natural isotopic mixtures (Hartmann et al. 2007). The high scattering power of deuterium for neutrons compared to $\mathrm{X}$-rays is, however, an important tool to determine the correct orientation of $\mathrm{BD}_{4}$ groups and their bonding schemes with the cations.

\section{In-situ synchrotron radiation powder X-ray diffraction (SR-PXD)}

The characterization of borohydrides is typically done by the powder X-ray diffraction (PXD), in conjunction with spectroscopic methods like Raman, infrared and NMR spectroscopy as well as thermal desorption studies. The synchrotron X-ray powder diffraction (SR-PXD) is nowadays routinely used to study light metal borohydrides (Filinchuk et al., 2010a), typically exploiting an area detector and Debye-Scherrer geometry (Fig. 2). The advantage of this setup, which enables in-situ studies, is the simple sample environment and fast data collection, high data collection rates, excellent time resolution, highly accurate measurements of diffracted intensities (good powder average), and a lower sensitivity to preferred orientation

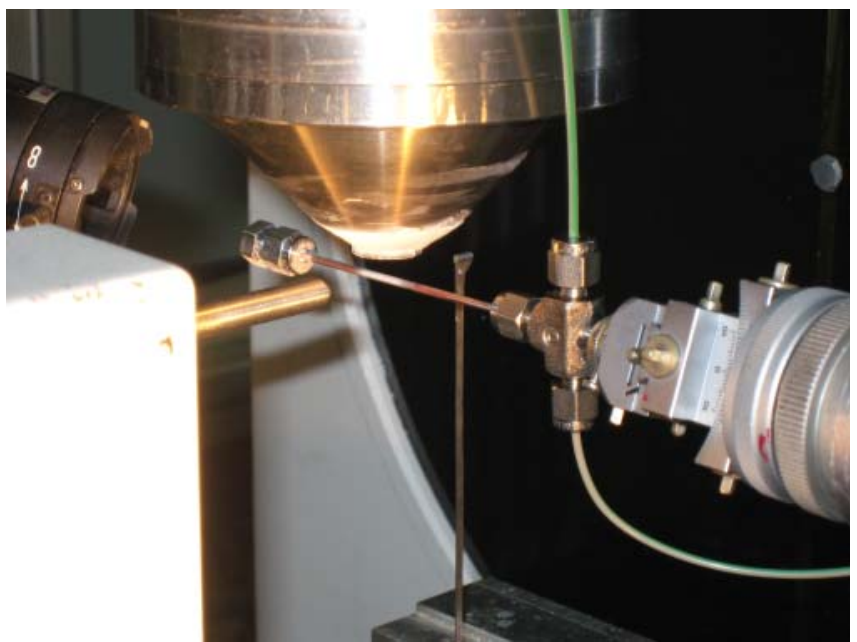

Fig. 2. A typical experimental set-up for the in-situ studies of light metal hydrides using an area detector and a Debye-Scherrer geometry.

effects. A combination of high counting rates with excellent reciprocal space resolution provided by curved solid state detectors (Mozzanica et al., 2009) enables in-situ study of even more complex structures.

In order to study the thermal decomposition of borohydrides, it is necessary to perform in-situ time- and temperature-resolved experiments. X-ray scattering typically requires a shorter data acquisition time compared to neutron diffraction and is therefore more amenable both to study fast reactions involving metastable compounds and intermediate decomposition products and also for kinetic studies of hydrogen absorption and desorption. Thinwalled glass capillaries commonly used as sample holders give a relatively high amorphous background and react with some borohydrides at elevated temperatures (Mosegaard et al., 2008). Single-crystal sapphire capillaries are an option to remove both undesired effects (Chupas et al., 2008; Jensen et al., 2010). Strong diffraction spots from the sapphire should be masked when using 2D detectors. Thin-walled glass and single-crystal sapphire capillaries enable in-situ experiments under hydrogen gas pressures of up to 100 bar and 300 bar, respectively (Jensen et al., 2010). An externally calibrated gas blower (Fig. 2) or a cryostream are used to control the temperature, while a filament heater is combined with a thermocouple placed inside a sapphire capillary next to the sample. The sapphirebased cell can be connected to the hydrogen source via both ends, thus preventing sample displacement even during rapid pressure changes. The thin-walled capillary setup can be combined with a gas system equipped with slowacting valves (Llewellyn et al., 2009), enabling pressure variation speeds of up to 100 bar per minute.

\section{$A b$-initio structure determination}

The diffraction methods for structural characterization of metal hydrides have been recently reviewed by Černý, 2008. Here we complement it with a few points specific to metal borohydrides, or advanced in the course of their investigation. 


\section{Indexing and space group determination}

The bottle-neck of the ab-initio structural studies of new compounds is indexing the powder patterns, which is extremely challenging for multiphase samples prepared by the ball milling. A successful strategy consists of the so-called "decomposition-aided indexing", which utilizes in-situ diffraction as a function of temperature (which is typically linearly dependent on time, this mode is known as "T-ramping") up to the decomposition/melting temperature of different phases. This procedure enables to separate diffraction peaks of individual phases as illustrated in Fig. 3 by SR-PXD for a ball milled $\mathrm{NaBH}_{4}-\mathrm{AlCl}_{3}$ mixture (Lindemann et al., 2011).

In this system a ball-milled sample contains one new phase, $\mathrm{NaAl}\left(\mathrm{BH}_{4}\right)_{4}$, two solid solutions $\mathrm{Na}\left(\mathrm{BH}_{4}, \mathrm{Cl}\right)$ and $\mathrm{Na}\left(\mathrm{Cl}, \mathrm{BH}_{4}\right)$, and two uknown impurities 1 and 2. Only $T$-ramping has enabled the assignment of observed peaks to individual phases, and led to a successful indexing of powder patterns. In order to eliminate an uncertainty introduced by a non-ideal centering of a capillary, it should be rotated within the same angular range during exposures, providing a smooth variation of the cell parameters. Useful guidelines for successful indexing of powder data can be found in David and Shankland, 2008, and Černý and Favre-Nicolin, 2007.

Space group determination can be another critical point: "While observing lattice-centering extinctions is usually relatively easy, the determination of the correct space group symmetry elements is generally more challenging" (David and Shankland, 2008). This is even more valid for poorly crystallized hydrides with broad peaks and strong pseudosymmetry, which is difficult to detect in an X-ray pattern due to the weak X-ray scattering power of hydrogen. Thus, careful examination of various space groups is warranted, first of all by comparing the results of the re-

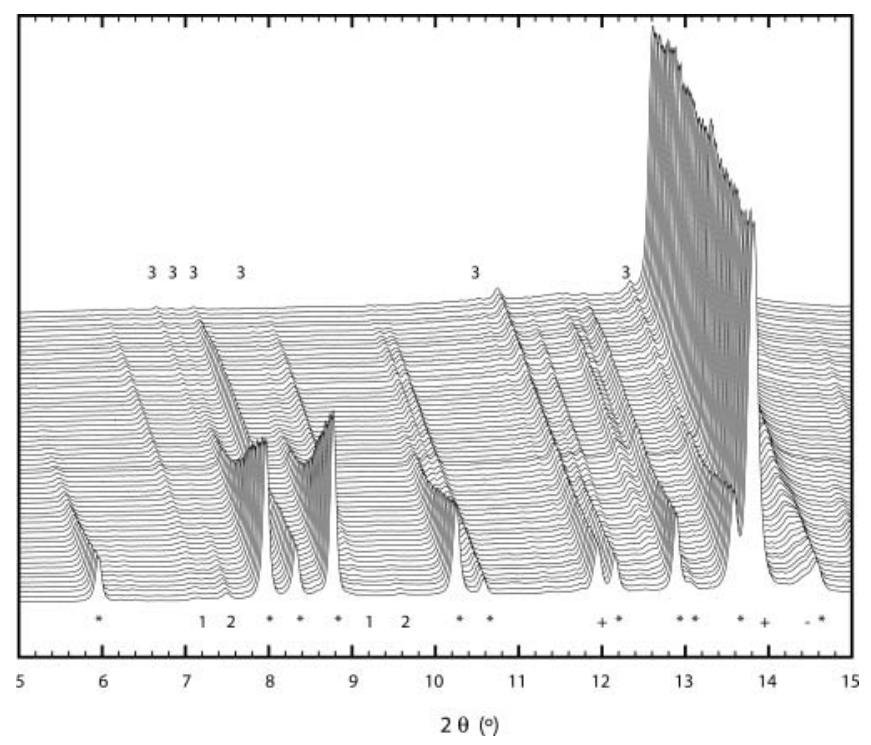

Fig. 3. In-situ synchrotron radiation $\mathrm{X}$-ray powder diffraction data for a ball milled sample of $\mathrm{NaBH}_{4}-\mathrm{AlCl}_{3}(3: 1)\left(62\right.$ to $125^{\circ} \mathrm{C}$, $\lambda=0.7296 \AA)$. Only utilization of $T$-ramping enabled attribution of observed peaks to individual phases, and led to a successful indexing of the powder patterns. Symbols: $* \mathrm{NaAl}\left(\mathrm{BH}_{4}\right)_{4},+\mathrm{Na}\left(\mathrm{BH}_{4}, \mathrm{Cl}\right)$, $-\mathrm{Na}\left(\mathrm{Cl}, \mathrm{BH}_{4}\right), 1$ and 2 unknown impurities, $3 \mathrm{NaAlCl}_{4}$ (Lindemann et al., 2011). spective Le Bail fits. The program ChekCell (Laugier and Bochu, 2004) helps to find the correct space group by an automated analysis of the systematic absences. Its subroutine TrueCell enables identifying higher metric symmetry consistent with a list of indexed peaks, and most importantly makes the search for various supercells taking into account less reliable or weak peaks put aside during indexing. Finally, the space group search can be easily made by attempting structure solution in different space groups. This is easily done when using the structure solution program Fox (Favre-Nicoline and Černý, 2002): going trough the space groups with the same number of independent units does not require any change in the input. Structure solution can be accomplished in lower symmetry space groups, down to $P 1$, using Fox and structural information expressed via constraints/restraints or using the program Superflip (Palatinus and Chapuis, 2007) on high-resolution powder data. The true higher symmetry of the resulting structures can be determined by automatic procedures implemented in Platon (Spek, 2006) and Superflip.

\section{Need for precise unit cell content?}

Direct space modeling of relatively complex structures can yield a relatively good fit for a wrong structure or even for a wrong chemical composition. The recently discovered borohydrides $A \mathrm{Zn}_{2}\left(\mathrm{BH}_{4}\right)_{5}(A=\mathrm{Li}, \mathrm{Na})$ (Ravnsbæk et al., 2009) are an example how important is the correct estimation of the unit cell content of an unknown phase. In a parallel study (Černý et al., 2010a) the chemical composition of the ball milled product was wrongly estimated as $A \mathrm{Zn}\left(\mathrm{BH}_{4}\right)_{3}$, and led to structural models that have shortcomings in Rietveld plots (Fig. 4) which are not easily detectable from the low resolution powder diffraction data (weakly diffracting and badly crystallized compound). Such a structural model can have balanced interatomic forces as shown by DFT optimization, but does not correspond to a stable polymorph at given thermodynamic conditions. The only warning about the wrong structural model came from the significant increase of the cell volume $(+5.2 \%)$ in the DFT optimized structure, because of higher cell content

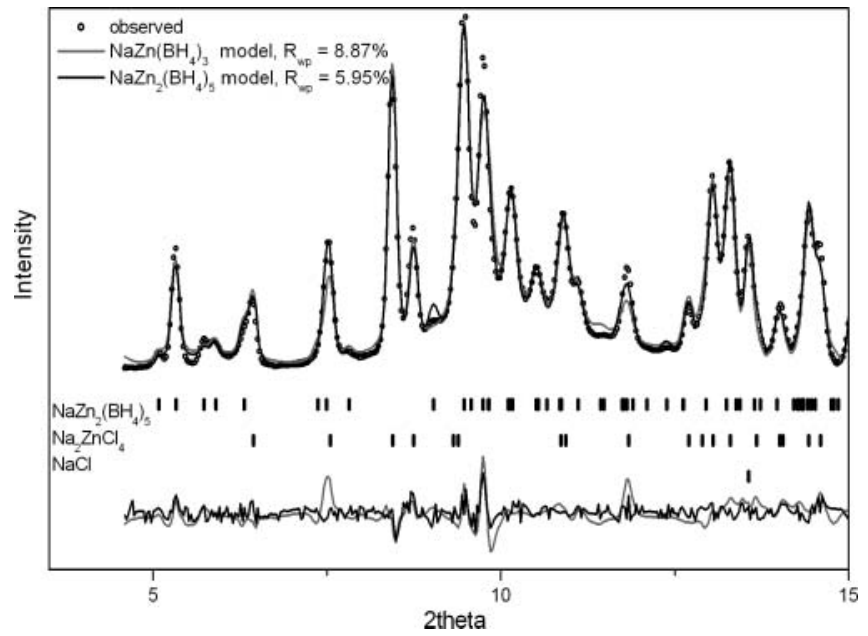

Fig. 4. Comparison of Rietveld plots for wrong, $\mathrm{NaZn}\left(\mathrm{BH}_{4}\right)_{3}$, and correct, $\mathrm{NaZn}_{2}\left(\mathrm{BH}_{4}\right)_{5}$, structural models (Černý et al.; 2010a), both in the same monoclinic cell (e.g. $P 2_{1} / c, a=9.440(4), b=16.573(4)$, $\left.c=9.110(2) \AA, \beta=112.99(3)^{\circ}\right)$. 
(16 cations and 24 anions) compared to the correct model (12 cations and 20 anions). It means also that the wrong model is more dense $\left(34.52 \AA^{3} /\right.$ ion) than the correct one (40.81 $\AA^{3} /$ ion) as calculated from DFT-optimized cells. Another warning for the wrong model could have been certainly obtained from the measured density, a difficult experiment for a relatively reactive powder.

It is interesting that $A \mathrm{Zn}\left(\mathrm{BH}_{4}\right)_{3}$ exists for $A=\mathrm{Na}$, though with different symmetry and structure (for more details see Černý et al., 2010a). The difference of DFT total energies (neglecting zero point energies) between the stable and hypothetic polymorphs of $\mathrm{NaZn}\left(\mathrm{BH}_{4}\right)_{3}$ is merely $0.151 \mathrm{eV} /$ f.u. $(=14.6 \mathrm{~kJ} / \mathrm{mol})$, in favor of the observed structure. There is no distinct structural relation between the two polymorphs of $\mathrm{NaZn}\left(\mathrm{BH}_{4}\right)_{3}$. Once the composition is known, crystal structure prediction can help to solve a structure from poor experimental data, despite theory does not necessarily yield the most stable structure (Roy and Matzger, 2009).

In the case of multiphase samples containing new phase with unknown exact chemical composition it is therefore crucial to perform the synthesis of starting mixtures in several different ratios of the starting compounds. This procedure enables deducing the chemical composition of the new phase from molar fractions of known phases in different mixtures. It is worth to finally note that the structure solution in Fox versus high quality data can reduce the number of atoms in order to reach the correct composition: provided the atoms types are correct and given in excess to the expected composition, the structure will be solved, and the excess atoms will be eliminated by using so called "Dynamic Occupancy Correction" (Favre-Nicolin and Černý, 2002).

\section{Direct- or reciprocal-space method?}

Crystal structures of light metal hydrides may be solved by any of the methods optimized for working with powder diffraction data (David et al., 2002). The methods requiring the extraction of structure factor amplitudes like the reciprocal (RSM) or dual space (for example charge flipping, Baerlocher et al., 2007) methods suffer from peak overlap in a powder pattern, and are less often used, especially for ball milled samples (Černý, 2008). Significant progress in the intensity partitioning for overlapping peaks was achieved by employing an additional parameter like temperature (anisotropic thermal expansion) or preferred orientation (see David and Shankland, 2008 for more details). The principal advantage of the direct space methods (DSM) is working without structure amplitudes extraction, thus being able to work with lower resolution powder data (Černý, 2008).

An excellent example when both, RSM and DSM, are equally suitable to solve a complex structure from high resolution powder data, is $\alpha-\mathrm{Mg}\left(\mathrm{BH}_{4}\right)_{2}$ (Fig. 5).

It represents one of the most complex atomic arrangements ever solved from powder diffraction data $\left(a=10.3182, c=36.9983 \AA\right.$, space group $\left.P 6_{1}\right)$. It was solved independently by DSM (Ćerný et al., 2007) and by RSM (Her et al., 2007). In both cases high resolution synchrotron data were required for indexing. In the DSM

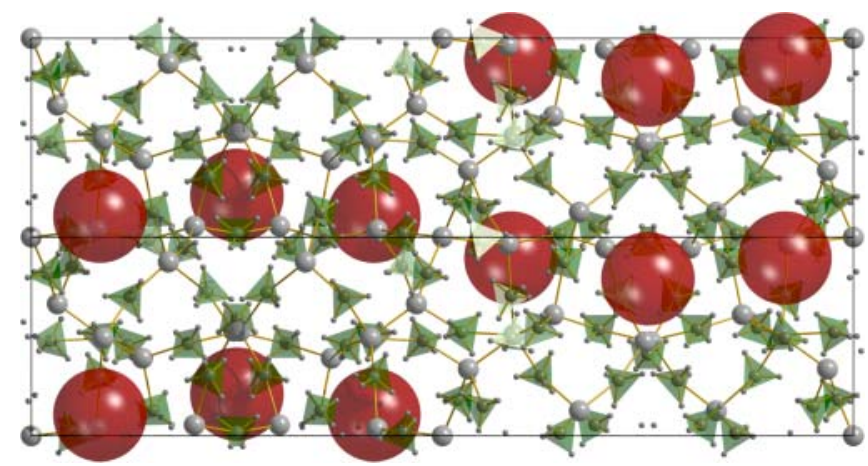

Fig. 5. Crystal structure of $\alpha-\mathrm{Mg}\left(\mathrm{BH}_{4}\right)_{2}$, first solved from SR-PXD data by direct- and reciprocal-space methods (Černý et al., 2007; Her et al., 2007) and then revisited by single-crystal diffraction (Filinchuk et al., 2009a). The latter enabled identifying the unoccupied voids shown as large spheres.

approach, the structure was modeled by five free $\mathrm{Mg}$ atoms and ten rigid tetrahedral $\mathrm{BD}_{4}$ groups, and optimized jointly against synchrotron and neutron data by using Fox. The correct structure was recognized after several hours of optimization. In the RSM approach, the structure was solved with the aid of the program EXPO (Altomare et al., 2004) using only synchrotron data. The positions of $\mathrm{Mg}$ and $\mathrm{B}$ atoms were recognized in electron density maps. In both cases the structure was refined by Rietveld method keeping the borohydride groups rigid.

Fox (Favre-Nicoline and Cerný, 2002) became the most popular direct space program in the "hydride world". The complexity of the structures solved by this program ranges from 2 to 55 independent atoms. Guidelines for efficient use of Fox can be found in Černý and Favre-Nicolin, 2007.

For the successful structure solution from textured powder samples, for instance phases forming in a diamond anvil cell (DAC), it is essential to take into account the preferred orientation. In Fox, it can be directly modelled on the stage of structure solution, by including one parameter (March-Dollase model) into the global optimization. Various directions of the preferred orientation should be tested independently, e.g. for the orthorhombic cell the three main axes should be tested as directions of the preferred orientation. In a typical high-pressure experiment, the compression direction coincides with the direction of the primary beam. Than, the Debye rings appear with a regular intensity distribution, and the texture is visually undetectable even with an area detector. Thus, when all attempts to solve a structure from high-pressure data fail, the texture can be assumed a priori, making it a part of the globally optimized model (see an example in Filinchuk et al., 2007). Certainly, simultaneous solution of an unknown structure and texture is the option of the last resort. There is another approach (Whitfield, 2009) to account for texture during the simulated annealing using the program Topas (Coelho, 2004). It uses spherical harmonics preferred orientation correction and does not require an input of a direction of the preferred orientation.

\section{Structure refinement by Rietveld method}

Even if other excellent Rietveld codes like GSAS [Larson and Von Dreele, 1994] are available, two programs are pre- 
ferred by the hydrogen community: Fullprof (RodríguezCarvajal, 1993) and Topas (Coelho, 2004). Both allow for joint refinement of several powder patterns, rigid body modeling, anisotropic line broadening modeling (even if not often observed in borohydrides) and sequential refinement (see below), i.e. automatic refinement of a structure vs. many powder patterns collected as a function of the temperature or pressure.

Typically the structures of borohydrides are solved and refined with the $\mathrm{BH}_{4}$ groups defined as rigid or semi-rigid ideal tetrahedra with one common refined $\mathrm{B}-\mathrm{H}$ distance. For the $\mathrm{BH}_{4}$ groups situated on a symmetry element the $\mathrm{BH}_{4}$ tetrahedra are allowed only to translate and to rotate following the operations of the symmetry element. The displacement parameters are typically refined isotropically.

Hints for Fullprof: Chemical information expressed in a form of soft restrains on bond distances and angles is taken as additional observations, weighted by the user-defined standard uncertainties. The list of possible restrains is automatically generated within the maximal distance limits Max_dst and stored in the .tpcr file. Restraints for the torsion angles can not be defined explicitly, but only via 1,4-distances. The hard constraints are neither available, but a similar result can be achieved by reducing the estimated uncertainties, thus imposing "very hard restraints". This leads to oscillations in the least-square refinement, which can be partly suppressed by using dumping factors (relaxation factors for shifts) for atomic positions, i.e. by reducing $R \_$at from 1 to 0.5 or less.

The absence of explicit constraints for bond distances and obstacles in defining torsion angles is a major drawback for refining large (organic) molecules in Fullprof. On the other hand, hydride structures usually contain much smaller rigid units, such as the tetrahedral $\mathrm{BH}_{4}$, which can be parameterized relatively well by restraints, as well as by using rigid body formalism $(J d i=4)$. The latter works with spherical internal coordinates and six additional parameters defining the group's position and orientation.

The spherical coordinates $(r, \varphi, \theta)$ of a rigid body are calculated from the crystallographic coordinates $(x, y, z)$ in Fullprof by the rigid body routine. The consistent atoms must have their atomic crystallographic coordinates $(x, y, z)$ chosen among the equivalent coordinates of a Wyckoff site so, that they are in contact and make a complete molecule. The best way to achieve this is to export coordinates from Fox as a CIF file. At the end of the rigid body refinement in Fullprof the crystallographic coordinates $(x, y, z)$ with their uncertainties can be obtained by exporting the results to an Ortep file (created automatically) or to a Shelx file $(F o u=2)$.

The use of rigid body parametrization enables to reduce a number of free coordinate parameters, and in case of a pronounced molecular libration to approximate better the atomic displacements. For that, a TLS formalism (Schomaker and Trueblood, 1968), which parametrizes vibrational motions or orientational disorder of a rigid-body object by a translational (T), librational (L), and screw (S) tensors, is implemented in Fullprof ( furth $=21$ ). Analysis of single crystal and powder synchrotron diffraction data on the high-temperature $\mathrm{LiBH}_{4}$ phase (Filinchuk et al., 2008a) consistently determined a significant disorder of the $\mathrm{BH}_{4}$ group. It is remarkable that, under reasonable rigid body assumptions, anisotropic atomic displacement parameters can be extracted for the $\mathrm{BH}_{4}$ group (in TLS model) and for the Li atom (2-rank tensor) from powder diffraction data collected at a synchrotron source. On the contrary, data of limited quality (e.g. collected in DACs) or for compositions containing heavy atoms allow only refinement of the rigid groups, while their orientation is determined from geometrical considerations (Černý et al., 2009; Filinchuk et al., 2010b) or DFT calculations (Černý et al., 2010a; Filinchuk et al., 2009c) etc.

Hints for Topas: Topas program enables easy definition of rigid bodies by a macro language; the most common objects like tetrahedron or octahedron are available already as macros provided with the program. The uncertainties of crystallographic coordinates of hydrogen atoms refined in Topas as a part of a rigid body are not available from the least squares matrix, but can be estimated by bootstrapping (Efron and Tibshirani, 1986).

The use of antibump restraints for $\mathrm{H}-\mathrm{H}$ and cation- $\mathrm{H}$ distance helps to find an orientation of $\mathrm{BH}_{4}$ groups and to stabilize the rigid body refinement. Examples of reliable structure models refined without restraints are also known (Černý et al., 2010b). Nevertheless, minimization of short repulsive interatomic contacts helps to construct a reasonable structural model. For instance, orientation of the neighbouring $\mathrm{BH}_{4}$ groups in the complex anions $\left[\mathrm{Sc}\left(\mathrm{BH}_{4}\right)_{4}\right]^{-1}$ in alkali metal-scandium borohydrides (Černý et al., 2010b, c) can be determined by excluding configurations with unreasonably short $\mathrm{H}-\mathrm{H}$ distances. Another example when a "tight space" was exploited in structure solution is the HTphase of $\mathrm{Y}\left(\mathrm{BH}_{4}\right)_{3}$ (Ravnsbæk et al., 2010b), where yttrium atom is octahedraly coordinated by the borohydride groups.

A note should be made on the accuracy and precision of the diffraction data and their influence on the refined parameters. An analysis of intensity distributions along the Debye rings suggests that the powder data obtained with 1D detector suffer from a poor powder average that may result in spuriously deformed $\mathrm{BH}_{4}$ tetrahedron (Filinchuk et al., 2008a). Intensity integration over the Debye rings recorded with a $2 \mathrm{D}$ detector provides much better average even for grainy sample and therefore higher reliability of the refined structural parameters. Accurate data normally yield the ideal geometry of the tetrahedral $\mathrm{BH}_{4}$ units (Filinchuk et al., 2008b).

The extremely high counting statistics allows reliable refinement of the light metal borohydrides structures, despite their low scattering power (Fig. 6). Proper modeling of the background, often appearing high relative to the weak diffraction peaks, enables to minimize the bias in the refined structural parameters. The precise modeling of the background is naturally extremely important. Two methods provide particularly good results: Fourier filtering in FullProf $(N b a=-2)$ and modeling with Chebyshev polynomials in Topas.

In order to obtain unbiased refined parameters, the data should also be correctly weighted in the least-square refinement by Rietveld method. For this, the $2 \theta$ - Intensity data obtained from an integration of the 2D rings should be complemented by a third column containing uncertainties of the integrated intensities, $\sigma(I)$, calculated consider- 


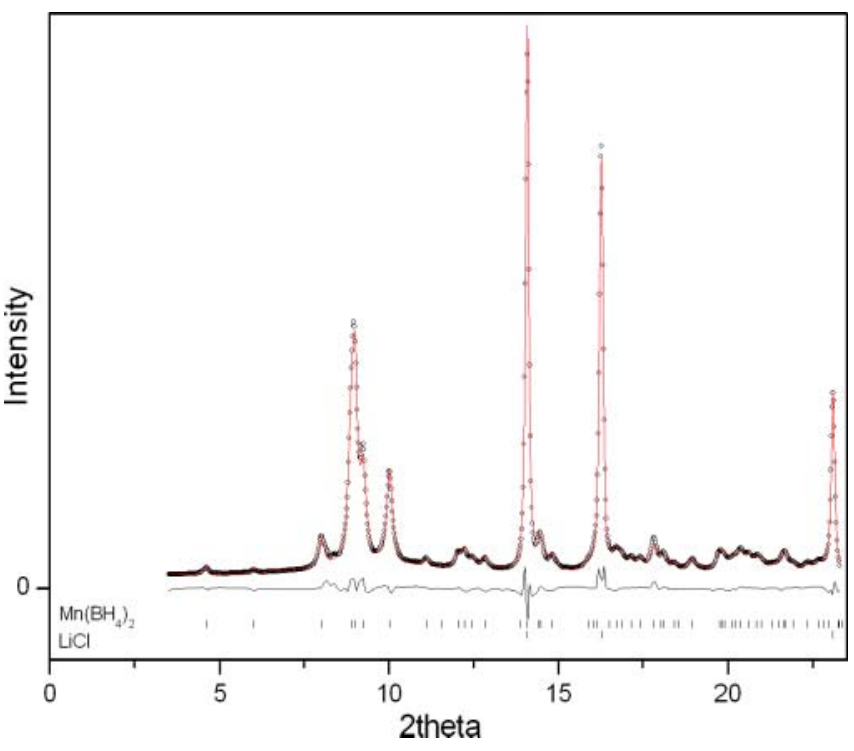

Fig. 6. Rietveld plot of $2 \mathrm{LiBH}_{4}: \mathrm{MnCl}_{2}$ mixture showing good modeling of weak Bragg peaks with synchrotron data from 2D-detector with high counting statistics (Černý et al., 2009).

ing the Poisson statistics of the real photon counts (Vogel et al., 2002). The use of the three-column data format slightly improves the quality of structural parameters, and yields large values of $\chi^{2}$. The latter underlines a statistically large difference between the observed and theoretical patterns in the presence of a vanishingly small noise and thus should not be considered as a warning in the refinement versus area detector powder data.

\section{Structure validation and analysis}

Structural information obtained from a Rietveld refinement can be augmented in a number of ways: by using diffraction data collected from the same phase at different conditions, by optimizing the structural model by DFT methods, by comparing the new structure with others members of a family. Besides bringing new information on stability and structural evolution of the phase of interest, this type of analysis helps to validate the structure or to reveal and resolve inconsistencies undetectable in the course of a routine Rietveld refinement based on a single dataset.

If a chemical reaction takes place during an in-situ study, we recommend first to compare the nominal composition of the starting bulk sample with the refined composition of multiphase product, involving new phases. Any discrepancy will either point to an error in the stoichiometry of the new structure or to a formation of an amorphous phase (often visible from an appearance of a wavy background).

\section{Exploiting fully in-situ powder diffraction data}

Besides the "decomposition-aided indexing" mentioned above, the vast data collected as a function of temperature $T$ or pressure $P$ enables to follow the structure evolution within its stability $P T$-region. A standard Fullprof input .pcr file can be used in so-called "sequential refinement", when the result of a converged Rietveld refinement from one pattern is used as a starting point of the refinement with the next pattern. This procedure is repeated cyclically, thus enabling to retrieve temperature and pressure dependences of various structural and profile parameters. A fine slicing of $T$ and $P$ helps to keep this process stable and enables detection of even small relative variations, which in turn shed light on bonding schemes and phase transition mechanisms. An input file should allow refining the background which usually varies during in-situ measurements, as well as allow for sufficient number of least-square cycles to achieve a convergence on every dataset. Global parameters, which are expected to be stable during the whole measurement, such as zero shift, should be refined from the first pattern, and the obtained value fixed in the sequential refinement. It is clear, that the correct structural model should fit well all the available data in the phase stability $P T$-region.

A few light hydride systems have been studied by Rietveld methods as a function of temperature and/or pressure: these are $\mathrm{LiBH}_{4}$ (Filinchuk et al., 2008a, c; Dmitriev et al., 2008; Hagemann et al., 2009), $\operatorname{Mg}\left(\mathrm{BH}_{4}\right)_{2}$ (Filinchuk et al., 2009a; Riktor et al., 2007), $\mathrm{Ca}\left(\mathrm{BH}_{4}\right)_{2}$ (Filinchuk et al., 2009b; Riktor et al., 2007), $\mathrm{NaBH}_{4}$ (Kumar and Cornelius, 2005; Babanova et al., 2009), $\mathrm{RbBH}_{4}$ (Filinchuk et al., 2010b), $\mathrm{NH}_{3} \mathrm{BH}_{3}$ (Filinchuk et al., 2009c) etc. Synchrotron radiation was used in all cases, enabling rapid data collection with a fine steps in $P$ and $T$. Exhaustive data analysis yielded a picture of structural evolution expressed in terms of order parameters and uncovered the $P-T$ phase diagrams.

A symmetry-based analysis of the structures observed under various $P-T$ conditions helps to understand atomistic mechanisms and thermodynamic aspects of the phase transitions. The analysis based on Landau theory, combined with the powder diffraction experiment, enables to construct a phenomenological pressure-temperature phase diagram, and to predict new polymorphs, as for example for a low- $T$ high- $P$ phase of $\mathrm{NH}_{3} \mathrm{BH}_{3}$ (Filinchuk et al., 2009c).

\section{Post-experimental evaluation}

A post-experimental DFT-optimization helps to validate new structures, locate light hydrogen atoms, especially when using high-pressure diffraction data of limited quality (Filinchuk et al., 2008c; Filinchuk et al., 2009c), and even to correct the symmetry and some structural details:

In the case of $\mathrm{Li}_{4} \mathrm{Al}_{3}\left(\mathrm{BH}_{4}\right)_{13}(P-43 n$, own structure type) the DFT optimization of the experimental structure has corrected the orientation of the complex anion $\left[\mathrm{Al}\left(\mathrm{BH}_{4}\right)_{4}\right]^{-}$, thus revising the originally anticipated bonding scheme implicating isolated complex ions $\left[\left(\mathrm{BH}_{4}\right) \mathrm{Li}_{4}\right]^{3+}$ and $\left[\mathrm{Al}\left(\mathrm{BH}_{4}\right)_{4}\right]^{-}$to a 3D-framework structure (Fig. 7). The DFT-optimized experimental model has shown an improved fit to the experimental data (Lindemann et al., 2010).

One of the complex cases, where theoretical methods contributed to the final structure determination is $\alpha$ $\mathrm{Mg}\left(\mathrm{BH}_{4}\right)_{2}$ :

The difficulty in finding the correct orientation of $\mathrm{BH}_{4}$ tetrahedra led to overlooking 2-fold axes in the $\alpha$ - 

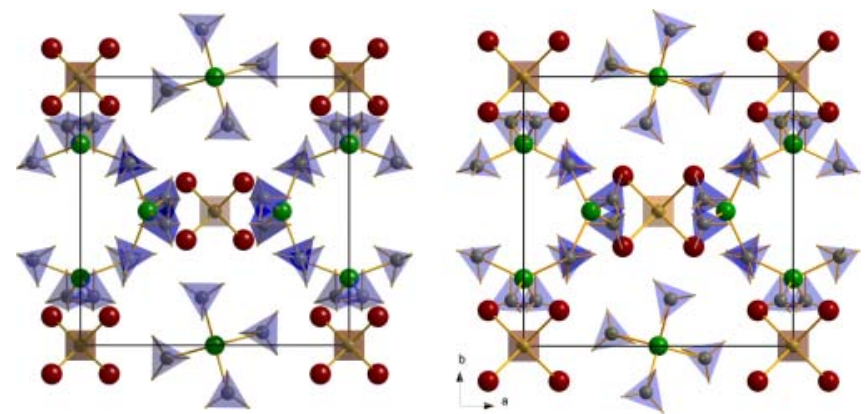

Fig. 7. Unit cell of $\mathrm{Al}_{3} \mathrm{Li}_{4}\left(\mathrm{BH}_{4}\right)_{13}$ as solved from SR-XPD (left) and as corrected by the DFT optimization (right). Complex cation $\left[\left(\mathrm{BH}_{4}\right) \mathrm{Li}_{4}\right]^{3+}$ centred by $\mathrm{BH}_{4}$ tetrahedron (brown), complex anion $\left[\mathrm{Al}\left(\mathrm{BH}_{4}\right)_{4}\right]^{-}$centred by $\mathrm{Al}$ atom (green).

$\operatorname{Mg}\left(\mathrm{BH}_{4}\right)_{2}$ structure model, and the space group symmetry was originally identified as $P 6_{1}$ (Černý et al., 2007; Her et al., 2007). The DFT optimization of the experimental model (Dai et al., 2008) has suggested space group $P 6_{1} 22$ as a possible true symmetry, which was later unambiguously confirmed by the single crystal X-ray diffraction (Filinchuk et al., 2009a). The fact that the structural solution has been found independently by DSM and RSM in the lower Laue symmetry $6 / m$ (space group $P 6_{1}$ ) rather than in the true Laue symmetry $6 / \mathrm{mmm}$ (true space group $\left.P 6_{1} 22\right)$ can be explained by the use of twice as many degrees of freedom in the former case, taking fully overlapping $h k l$ and $k h l$ reflections as fully independent, thus allowing to compensate for systematic errors. Single-crystal diffraction, where these reflections are measured separately, is more stable with respect to this bias. It is interesting that ADDSYM procedure in Platon (Spek, 2006) did not detect higher symmetry. Analysis of the published $P 6_{1}$ model shows that location of the H-atoms from powder data posed the main problem for the identification of the correct symmetry: wrongly determined orientations of some $\mathrm{BH}_{4}$ groups hampered a successful detection of the true $P 6_{12} 22$ symmetry by automatic algorithms. A DFT optimization easily gets away from the local minimum of the experiment cost-function. Since Rietveld refinement and the DFT method are based on two very different cost functions, their combination greatly helps to reach a global minimum. This is especially important for hydrides, since the experimental data are less sensitive to the positions of hydrogen atoms. We can therefore highly recommend post-experimental DFT optimization of crystal structures for the systems containing light elements such as hydrogen.

When atomic positions are roughly correct, a possibly higher crystallographic symmetry of a structure model can be detected by the ADDSYM routine. Often it is easier to solve a structure in a lower symmetry and then find the missing elements by this automatic routine. For instance, such a situation took place during the structure solution of $\mathrm{Mn}\left(\mathrm{BH}_{4}\right)_{2}$ which was solved in the space group $P 3_{1}$ and than the true higher $P 3_{1} 12$ symmetry was identified in PLATON (Černý et al., 2009).

An automatic search for unoccupied voids (CALC VOID) in PLATON helps to reveal physically unrealistic cavities which usually indicate that some atoms are missing. Once the major part of the structure is identified, the structure can be completed using difference Fourier maps: they can be calculated both in Fox and in Fullprof. Amusingly, there are rare cases of truly empty voids in largely ionic borohydrides, see $\mathrm{Mg}\left(\mathrm{BH}_{4}\right)_{2}$ as an example (Fig. 5).

\section{Local structures and crystal chemistry}

A crystal chemistry analysis of a new hydride structure helps to position the given phase in the hierarchy of the known structure types and thus to conclude about the prevailing bonding. Good examples are alkali metal-scandium borohydrides (Hagemann et al., 2008; Černý et al., 2010b, c) where inorganic structure types based on packing of alkali metal cations and $\left[\mathrm{Sc}\left(\mathrm{BH}_{4}\right)_{4}\right]^{-1}$ anions $(h c p, f c c$ and $b c c)$ have been identified. Similarly, $\mathrm{Li}_{4} \mathrm{Al}_{3}\left(\mathrm{BH}_{4}\right)_{13}$ with its own structure type (Lindemann et al., 2010) can be rationalized as packing of $\left[\left(\mathrm{BH}_{4}\right) \mathrm{Li}_{4}\right]^{3+}$ and $\left[\mathrm{Al}\left(\mathrm{BH}_{4}\right)_{4}\right]^{-}$in the $\mathrm{Cr}_{3} \mathrm{Si}$ antitype, the latter is a Frank-Kasper phase. We will not cover the crystal chemistry of borohydrides here, as it was thoroughly reviewed by Filinchuk et al., 2008b; Filinchuk et al., 2010a and Ravnsbæk et al., 2010a. Instead we will point to a limitation of using experimental data and of the crystal chemistry background to resolve questions about local configuration in some hydride structures.

As already discussed for octahedraly coordinated yttrium in the HT-phase of $\mathrm{Y}\left(\mathrm{BH}_{4}\right)_{3}$, the X-ray powder diffraction may provide only an average structure without "seeing" properly the true local structure of possibly ordered hydrogen atoms. The HT-phase of $\mathrm{Y}_{(}\left(\mathrm{BH}_{4}\right)_{3}$ has been described from X-rays in the cubic cell $(P m \overline{3} m$, $a=5.4547(8) \AA$, Ravnsbæk et al., 2010b) containing $\mathrm{BH}_{4}$ tetrahedra rotationally disordered over two positions (Fig. 8a). Only when neutron powder diffraction data were measured the true cubic superstructure $(F m \overline{3} c, 2 a$, Frommen et al., 2010) with fully ordered $\mathrm{BH}_{4}$ tetrahedra has been discovered (Fig. 8b). Interestingly, the only superstructure reflection 531 accessible within a typical X-ray powder pattern (down to $d=1.4 \AA^{-1}$ ) has practically zero intensity, while it becomes the strongest reflection in the neutron powder pattern.

The non-periodic local ordering of $\mathrm{BH}_{4}$ groups producing a diffuse intensity in the powder pattern has not been observed yet in any known borohydride, even if it can be expected in disordered $R T$-cubic phases of alkali metal borohydrides $A \mathrm{BH}_{4}$ for $A=\mathrm{Na}, \mathrm{K}$ and $\mathrm{Rb}$. On the other hand the dynamic rotation disorder of the $\mathrm{BH}_{4}$ groups studied by various spectroscopic methods seems to be quite common feature of the alkali metal borohydrides as observed in HT-hexagonal $\mathrm{LiBH}_{4}$ (Skripov et al., 2008), RThexagonal $\alpha-\mathrm{Mg}\left(\mathrm{BH}_{4}\right)_{2}$ (Skripov et al., 2010) and RT-cubic phases of other alkali metal borohydrides (Verdal et al., 2010; Babanova et al., 2010). Freely rotating protons in the imide group $\mathrm{NH}$ have been proposed by molecular dynamics calculations in $\mathrm{Li}_{2} \mathrm{NH}$ (Ludueña and Sebastiani, 2010).

\section{Perspectives}

The importance of energy minimization like DFT calculation for the structure verification leads to the idea of active 

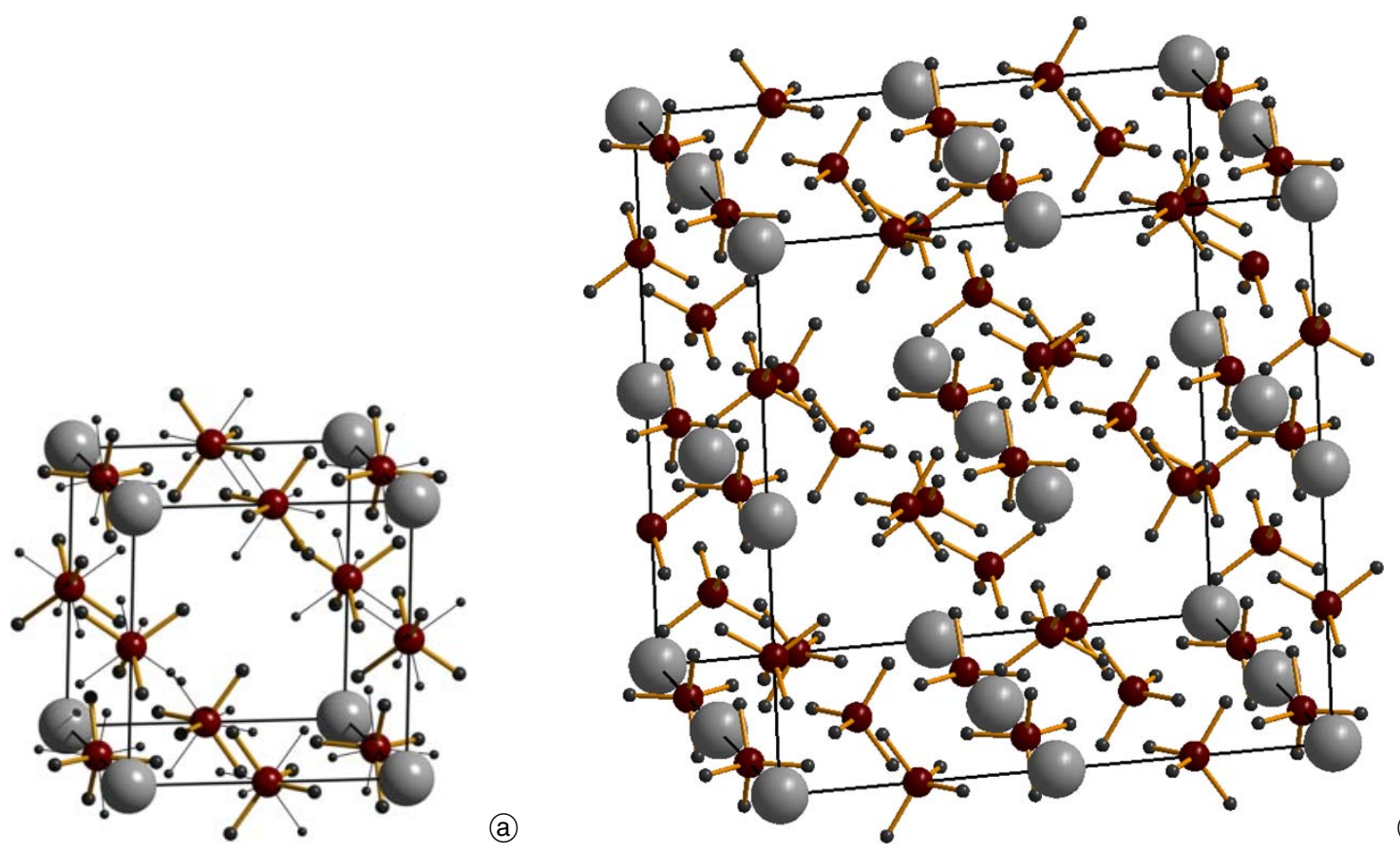

Fig. 8. Crystal structure of the high-temperature $\beta$ - $\left.\mathrm{Y}_{(\mathrm{BH}}\right)_{3}$ phase. (a) Two orientations of the disordered $\mathrm{BH}_{4}$ group in the structure as observed by $\mathrm{X}$-rays only are depicted by bold and thin lines: a possible local ordering of the $\mathrm{BH}_{4}$ groups, avoiding $\mathrm{H}-\mathrm{H}$ distances shorter than $2.48 \AA$, is shown. (b) The neutron powder diffraction data have confirmed this ordering scheme and revealed the superstructure with doubled cubic lattice parameter.

use of the energy minimization within the DSM structure solution. A simplified approach use the minimization of an empirical potential energy function appropriate to the system under study (Putz et al., 1999), and it has been successfully implemented for the inorganic structures in the programs Endeavour (Putz et al., 1999) and Topas (Coelho, 2004). In the case of light metal hydrides even more simplified method using only the anti-bump interatomic distances (the minimal distance between two atoms below which the actual structural configuration is highly penalized) as implemented in the program Fox (FavreNicoline and Černý, 2002) showed to be highly successful. Nevertheless, here discussed examples of $\mathrm{Mg}\left(\mathrm{BH}_{4}\right)_{2}$ and $\mathrm{Li}_{4} \mathrm{Al}_{3}\left(\mathrm{BH}_{4}\right)_{13}$ show that more precise energy minimization actively used within the DSM structure solution of light metal hydrides is highly desirable. Such procedures are known from crystal structure prediction like using more sophisticated energy landscapes of the chemical systems (Schön and Jansen, 2001; Woodley et al., 1999), free energy calculation (Glass et al., 2006) and DFT calculation (Shevlin and Guo, 2009). Even if the structure prediction of light metal hydrides based on the DFT calculations generally fails to predict the long range order (Hummelshøj et al., 2009), the same method jointly used with the powder diffraction data within the DSM algorithm will significantly increase the success rate of the structure solution process. The limiting factor seems to be still the available computing power.

Second significant improvement of the structural analysis of light metal hydrides waiting still for a broader use is so-called "parametric Rietveld refinement" (Stinton and Evans, 2007). It has not yet been used in studying the borohydrides if we do not count the parametrization of anisotropic line broadening like for example in the case of $\beta$ -
$\mathrm{Mg}\left(\mathrm{BH}_{4}\right)_{2}$ phase (Her et al., 2007). The parametric Rietveld refinement means constraining the crystallographic parameters (atomic coordinates, occupancies and displacement parameters) by set of equations which may have a physical basis, and enables more reliable and faster refinement providing directly some physical parameters like coefficients of thermal dilatation, rate constants for kinetic processes etc.

\section{Conclusions}

Structural characterization of light metal borohydrides is extremely challenging as these materials are often found as fine powders in synthesis products containing several compounds. New strategies for ab-initio structure solution had to be applied or developed, like synthesis with different ratio of starting products, 'decomposition-aided indexing', direct space methods guided by chemical information, structure verification by energy minimization. The time and temperature resolved in-situ powder X-ray diffraction data is the most important analysis technique capable of providing data for structural analysis, and giving detailed insight into the structural chemistry, and chemical reactions taking place during synthesis and decomposition. This new class of $\mathrm{BH}_{4}^{-}$based materials possesses the fascinating structural chemistry, exhibiting bonding ranging from ionic to more covalent with some degree of directionality in the $M-\mathrm{BH}_{4}$ interaction. Powder diffraction is likely our most valuable and versatile tool in order to discover and characterise novel materials, which can form the basis for new, sustainable and carbon free energy technologies. 
Acknowledgments. The authors thank to T. R. Jensen and all his group (University of Aarhus), especially D. B. Ravnsbaek for nice and fruitfull common work on borohydrides. The collaboration with D. Chernyshov and V. Dmitriev (SNBL at ESRF), H. Hagemann (University of Geneva), and N. Penin (CNRS and University of Bordeaux) is highly appreciated.

\section{References}

Adams, R. M.: Commission on nomenclature of inorganic chemistry of the inorganic chemistry division. Nomenclature of inorganic boron compounds. Pure Appl. Chem. 30 (1972) 681-710.

Altomare, A.; Caliandro, R.; Camalli, M.; Cuocci, C.; Giacovazzo, C.; Moliternia, A. G. G.; Rizzi, R.: Automatic structure determination from powder data with EXPO2004. J. Appl. Crystallography 37 (2004) 1025-1028.

Babanova, O.; Soloninin, A.; Stepanov, A.; Skripov, A.; Filinchuk, Y.: Structural and dynamical properties of $\mathrm{NaBH}_{4}$ and $\mathrm{KBH}_{4}$ : NMR and synchrotron X-ray diffraction studies. J. Phys. Chem. C. 114 (2010) 3712-3718.

Baerlocher, Ch.; McCusker, L. B.; Palatinus, L.: Charge flipping combined with histogram matching to solve complex crystal structures from powder diffraction data. Z. Kristallogr. 222 (2007) 47-53.

Balema, V. P.; Wiench, J. W.; Pruski, M.; Pecharsky, V. K.: Mechanically induced solid-state generation of phosphorus ylides and the solvent free Wittig reaction. J. Am. Chem. Soc. 124 (2002) 6244-6245.

Buchter, F.; Łodziana, Z.; Remhof, A.; Mauron, Ph.; Friedrichs, O.; Borgschulte, A.; Züttel, A.; Filinchuk, Y.; Palatinus, L.: Experimental charge density of $\mathrm{LiBD}_{4}$ from maximum entropy method. Phys. Rev. B. 83 (2011) 064107.

Černý, R.; Favre-Nicolin, V.: Direct space methods of structure determination from powder diffraction: principles, guidelines and perspectives. Z. Kristallogr. 222 (2007) 105-113.

Černý, R.; Filinchuk, Y.; Hagemann, H.; Yvon, K.: Magnesium borohydride: synthesis and crystal structure. Angew. Chem. Int. Ed. 46 (2007) 5765-5767

Černý, R.: Solving crystal structures of metal and chemical hydrides. Z. Kristallogr. 223 (2008) 607-616.

Černý, R.; Penin, N.; Hagemann, H.; Filinchuk, Y.: The first crystallographic and spectroscopic characterization of a 3d-metal borohydride: $\mathrm{Mn}\left(\mathrm{BH}_{4}\right)_{2}$. J. Phys. Chem. C. 113 (2009) 9003-9007.

Černý, R.; Kim, K. Ch.; Penin, N.; D’Anna, V.; Hagemann, H.; Sholl, D. S.: $A \mathrm{Zn}_{2}\left(\mathrm{BH}_{4}\right)_{5}(A=\mathrm{Li}, \mathrm{Na})$ and $\mathrm{NaZn}\left(\mathrm{BH}_{4}\right)_{3}$ : structural studies. J. Phys. Chem. C 114 (2010a) 19127-19133.

Černý, R.; Severa, G.; Ravnsbæk, D. B.; Filinchuk, Y.; d'Anna, V.; Hagemann, H.; Haase, D.; Jensen, C. M.; Jensen, T. R.: $\mathrm{NaSc}\left(\mathrm{BH}_{4}\right)_{4}$ : A novel scandium-based borohydride. J. Phys. Chem. C. 114 (2010b) 1357-1364.

Černý, R.; Ravnsbæk, D. B.; Severa, G.; Filinchuk, Y.; D’Anna, V.; Hagemann, H.; Haase, D.; Jensen, C. M.; Jensen, T. R.: Structure and characterization of $\mathrm{KSc}\left(\mathrm{BH}_{4}\right)_{4}$. J. Phys. Chem. C 114 (2010c) 19540-19549.

Coelho, A. A.: TOPAS-Academic (2004). http://members.optusnet.com. au/ alancoelho

Remark: Commercial version containing more comfortable GUI may be obtained from Bruker AXS.

Chupas, P. J.; Chapman, K. W.; Kurtz, C.; Hanson, J. C.; Lee, P. L.; Grey, C. P. J.: A versatile sample-environment cell for non-ambient X-ray scattering experiments. Appl. Cryst. 41 (2008) 822824.

Dai, B.; Sholl, D. S.; Johnson, J. K.: First-principles study of experimental and hypothetical $\mathrm{Mg}\left(\mathrm{BH}_{4}\right)_{2}$ crystal structures. J. Phys. Chem. C. 112 (2008) 4391-4395.

David, W. I. F.; Shankland, K.; McCusker, L. B.; Baerlocher, Ch.: Structure Determination from Powder Diffraction Data. Editors. Oxford University Press, 2002.

David, W. I. F.; Shankland, K.: Structure determination from powder diffraction data. Acta Cryst. A64 (2008) 52-64.

Dmitriev, V.; Filinchuk, Y.; Chernyshov, D.; Talyzin, A.; Dzwilewski, A.; Andersson, O.; Sundqvist, B.; Kurnosov, A.: Pressure-temperature phase diagram of $\mathrm{LiBH}_{4}$ : synchrotron X-ray diffraction experiments and theoretical analysis. Phys. Rev. B. 77 (2008) 174112.
Efron, B.; Tibshirani, R.: Bootstrap methods for standard errors, confidence intervals, and other measures of statistical accuracy. Stat. Science 1 (1986) 54-77.

Favre-Nicolin, V.; Černý, R.: FOX, "Free objects for crystallography": a modular approach to $a b$ initio structure determination from powder diffraction. J. Appl. Crystallography 35 (2002) 734-743. See also http://objcryst.sourceforge.net/Fox.

Filinchuk, Y.; Talyzin, A.; Chernyshov, D.; Dmitriev, V.: High-pressure phase of $\mathrm{NaBH}_{4}$ : crystal structure from synchrotron powder diffraction data. Phys. Rev. B. 76 (2007) 092104.

Filinchuk, Y.; Chernyshov, D.; Černý, R.: The lightest borohydride probed by synchrotron diffraction: experiment calls for a new theoretical revision. J. Phys. Chem. C. 112 (2008a) 10579-10584.

Filinchuk, Y.; Chernyshov, D.; Dmitriev, V.: Light metal borohydrides: crystal structures and beyond. Z. Kristallogr. 223 (2008b) 649-659.

Filinchuk, Y.; Chernyshov, D.; Nevidomskyy, A.; Dmitriev, V.: Highpressure polymorphism as a step towards destabilization of $\mathrm{LiBH}_{4}$. Angew. Chem. Int. Ed. 47 (2008c) 529-532.

Filinchuk, Y.; Černý, R.; Hagemann, H.: Insight into $\mathrm{Mg}\left(\mathrm{BH}_{4}\right)_{2}$ with synchrotron X-ray diffraction: structure revision, crystal chemistry, and anomalous thermal expansion. Chem. Mater. 21 (2009a) 925-933.

Filinchuk, Y.; Rönnebro, E.; Chandra, D.: Crystal structures and phase transformations in $\mathrm{Ca}\left(\mathrm{BH}_{4}\right)_{2}$. Acta Mater. 57 (2009b) 732-738.

Filinchuk, Y.; Nevidomskyy, A. H.; Chernyshov, D.; Dmitriev V. High-pressure phase and transition phenomena in ammonia borane $\mathrm{NH}_{3} \mathrm{BH}_{3}$ from X-ray diffraction, Landau theory, and ab initio calculations. Phys. Rev. B. 79 (2009c) 214111.

Filinchuk, Y.; Chernyshov, D.; Dmitriev, V.: Crystal chemistry of light metal borohydrides. In "Boron hydrides, high potential hydrogen storage materials", ed. U. B. Demirci and P. Miele, Nova Publishers (2010a); arXiv: abs/1003.5378.

Filinchuk, Y.; Talyzin, A. V.; Hagemann, H.; Dmitriev, V.; Chernyshov, D.; Sundqvist, B.: Cation size and anion anisotropy in structural chemistry of metal borohydrides. The peculiar pressure evolution of $\mathrm{RbBH}_{4}$. Inorg. Chem. 49 (2010b) 5285-5292.

Frommen, C.; Aliouane, N.; Daledda, S.; Fonneløp, J. E.; Grove, H.; Lieutenant, K.; Llamas-Jansa, I.; Sartori, S.; Sørby, M. H.; Hauback, B. C.: Crystal structure, polymorphism, and thermal proper-

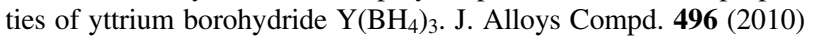
$710-716$.

Glass, C. W.; Oganov, A. R.; Hansen, N.: USPEX-Evolutionary crystal structure prediction. Comp. Phys. Comm. 175 (2006) 713-720.

Gray, E. Mac.; Cookson, D. J.; Blach, T. P.: X-ray diffraction cell for studying solid-gas reactions under gas pressures to 100 bar. J. Appl. Crystallogr. 39 (2007) 850-855.

Grochala, W.; Edwards, P. P.: Thermal decomposition of the non-interstitial hydrides for the storage and production of hydrogen. Chem. Rev. 104 (2004) 1283-1315.

Hagemann, H.; Longhini, M.; Kaminski, J. W.; Wesolowski, T. A.; Černý, R.; Penin, N.; Sorby, M. H.; Hauback, B. C.; Severa, G.; Jensen, C. M.: $\mathrm{LiSc}\left(\mathrm{BH}_{4}\right)_{4}$, a new complex salt with discrete $\mathrm{Sc}\left(\mathrm{BH}_{4}\right)_{4}^{-}$ions. J. Phys. Chem. A. 112 (2008) 7551-7555.

Hagemann, H.; Filinchuk, Y.; Chernyshov, D.; van Beek, W.: Lattice anharmonicity and structural evolution of $\mathrm{LiBH}_{4}$ : an insight from Raman and X-ray diffraction experiments. Phase Trans. 82 (2009) $344-355$.

Hagemann, H.; Černý, R.: Synthetic approaches to inorganic borohydrides. Dalton Trans. 39 (2010) 6006-60012.

Hartman, M. R.; Rush, J. J.; Udovic, T. J.; Bowman, R. C. Jr.; Hwang, S. J.: Structure and vibrational dynamics of isotopically labeled lithium borohydride using neutron diffraction and spectroscopy. J. Solid State Chem. 180 (2007) 1298-1305.

Hauback, B. C.: Structures of aluminium-based light weight hydrides. Z. Kristallogr. 223 (2008) 636-648.

Her, J.-H.; Stephens, P. W.; Gao, Y.; Soloveichik, G. L.; Rijssenbeek, J.; Andrus, M.; Zhao, J.-C.: Structure of unsolvated magnesium borohydride $\mathrm{Mg}\left(\mathrm{BH}_{4}\right)_{2}$. Acta Cryst. B. 63 (2007) 561-568.

Hummelshøj, J. S.; Landis, D. D.; Voss, J.; Jiang, T.; Tekin, A.; Bork, N.; Dułak, M.; Mortensen, J. J.; Adamska, L.; Andersin, J.: Density functional theory based screening of ternary alkali-transition metal borohydrides: A computational material design project. J. Chem. Phys. 131 (2009) 014101. 
James, B. D.; Wallbridge, M. G. H.: Metal tetrahydroborates. Prog. Inorg. Chem. 11 (1970) 99-231.

Jensen, T. R.; Nielsen, T. K.; Filinchuk, Y.; Jørgensen, J. E.; Cerenius, Y.; Gray, E. MacA.; Webb, C.: Versatile in-situ powder Xray diffraction cells for solid-gas investigations. J. Appl. Cryst. $\mathbf{4 3}$ (2010) 1456-1463.

Kumar, R. S.; Cornelius, A. L.: Structural transitions in $\mathrm{NaBH}_{4}$ under pressure. Appl. Phys. Lett. 87 (2005) 261916.

Larson, A. C.; Von Dreele, R. B.: General Structure Analysis System (GSAS). Los Alamos National Laboratory Report LAUR 86-748, 1994.

Laugier, J.; Bochu, B.: LMGP suite of programs for the interpretation of X-ray experiments. Laboratoire des Materiaux et du Génie Physique de l'Ecole Supérieure de Physique de Grenoble, 2004.

Lindemann, I.; Ferrer, R. D.; Dunsch, L.; Filinchuk, Y.; Černý, R.; Hagemann, H.; D’Anna, V.; Lawson Daku, L. M.; Schultz, L.; Gutfleisch, $\mathrm{O} .: \mathrm{Al}_{3} \mathrm{Li}_{4}\left(\mathrm{BH}_{4}\right)_{13}$ : a complex double-cation borohydride with a new structure. Chem. Eur. J. 16 (2010) 8707-8712.

Lindemann, I.; Ferrer, R. D.; Dunsch, L.; Černý, R.; Hagemann, H.; D’Anna, V.; Filinchuk, Y.; Schultz, L.; Gutfleisch, O.: Novel sodium aluminium borohydride containing the complex anion $\mathrm{Al}\left(\mathrm{BH}_{4}, \mathrm{Cl}\right)_{4}$. Faraday Discuss. 151 (2011) 231-242.

Llewellyn, P.; Horcajada, P.; Maurin, G.; Devic, T.; Rosenbach, N.; Bourrelly, S.; Serre, C.; Vincent, D.; Loera-Serna, S.; Filinchuk, Y.; Ferey, G. Complex adsorption of short linear alkanes in the flexible metal-organic-framework MIL-53(Fe). J. Am. Chem. Soc. 131 (2009) 13002-13008.

Ludueña, G. A.; Sebastiani, D.: Possibility of Coherent Delocalized Nuclear Quantum States of Protons in $\mathrm{Li}_{2} \mathrm{NH}$. J. Phys.Chem.Lett., 1 (2010) 3214-3218.

Marks, T. J.; Kolb, J. R.: Covalent transition metal, lanthanide, and actinide tetrahydroborate complexes. Chem. Rev. 77 (1977) 263-293.

Mosegaard, L.; Moeller, B.; Jorgensen, J.-E.; Filinchuk, Y.; Cerenius, Y.; Hanson, J.; Dimasi, E.; Besenbacher, F.; Jensen, T.: Reactivity of $\mathrm{LiBH}_{4}$ : in-situ synchrotron radiation powder X-ray diffraction study. J. Phys. Chem. C. 112 (2008) 1299-1303.

Mozzanica, A.; Bergamaschi, A.; Dinapoli, R.; Gozzo, F.; Heinrich, B.; Kraft, P.; Patterson, B.; Schmitt, B.; Mythen, H.; A 128 channel single photon counting readout chip. Nucl. Inst. Meth. A 607 (2009) 250-252.

Orimo, S.-I.; Nakamori, Y.; Eliseo, J. R.; Züttel, A.; Jensen, C. M.: Complex hydrides for hydrogen storage. Chem. Rev. 107 (2007) 4111-4132.

Palatinus, L.; Chapuis, G. Superflip - a computer program for the solution of crystal structures by charge flipping in arbitrary dimensions. J. Appl. Cryst. 40 (2007) 786-790.

Putz, H.; Schön, J. C.; Jansen, M.: Combined method for ab-initio structure solution from powder diffraction data. J. Appl. Cryst. 32 (1999) 864-870.

Ravnsbæk, D.; Filinchuk, Y.; Cerenius, Y.; Jakobsen, H. J.; Besenbacher, F.; Skibsted, J.; Jensen T. R.: A series of mixed-metal borohydrides. Angew. Chem. Int. Ed. 48 (2009) 6659-6663.

Ravnsbæk, D. B.; Filinchuk, Y.; Černý, R.; Jensen, T. R.: Powder diffraction methods for studies of borohydride-based energy storage materials. Z. Kristallogr. 225 (2010a) 557-569.
Ravnsbæk, D. B.; Filinchuk, Y.; Černý, R.; Ley, M. B.; Haase, D.; Jakobsen, H. J.; Skibsted, J.; Jensen, T. R.: Thermal polymorphism and decomposition of $\mathrm{Y}\left(\mathrm{BH}_{4}\right)_{3}$. Inorg. Chem. 49 (2010b) 3801-3809.

Riktor, M. D.; Sørby, M. H.; Chłopek, K.; Fichtner, M.; Buchter, F.; Züttel, A.; Hauback, B. C.: In situ synchrotron diffraction studies of phase transitions and thermal decomposition of $\mathrm{Mg}\left(\mathrm{BH}_{4}\right)_{2}$ and $\mathrm{Ca}\left(\mathrm{BH}_{4}\right)_{2}$. J. Mater. Chem. 17 (2007) 4939-4942.

Rodríguez-Carvajal, J.: Recent advances in magnetic structure determination by neutron powder diffraction. Physica B. 192 (1993) 55-69. Remark: For a more recent version see: Recent developments of the program FULLPROF. Newsletter of the Commission on Powder Diffraction (IUCr) 26 (2001) 12-19. The complete program and documentation can be obtained at http://www.ill.fr/ dif/Soft/fp/

Roy, S.; Matzger, A. J. Unmasking a third polymorph of a benchmark crystal-structure-prediction compound. Angew. Chem. Int. Ed. 48 (2009) 8505-8508.

Schomaker, V.; Trueblood, K. N.: On the rigid-body motion of molecules in crystals. Acta Crystallogr. B 24 (1968), 63-76.

Schön, J. C.; Jansen, M.: Determination, prediction, understanding of structures using the energy landscapes of chemical systems Parts I and II. Z. Kristallogr. 216 (2001) 307-325, 361-383.

Shevlin, S. A.; Guo, Z. X.: Density functional theory simulations of complex hydride and carbon-based hydrogen storage materials. Chem. Soc. Rev. 38 (2009) 211-225.

Skripov, A. V.; Soloninin, A. V.; Filinchuk, Y.; Chernyshov, D.: Nuclear magnetic resonance study of the rotational motion and the phase transition in $\mathrm{LiBH}_{4}$. J. Phys. Chem. C 112 (2008) 1870118705.

Skripov, A. V.; Soloninin, A. V.; Babanova, O. A.; Hagemann, H.; Filinchuk, Y. Nuclear magnetic resonance study of reorientational motion in $\alpha-\mathrm{Mg}\left(\mathrm{BH}_{4}\right)_{2}$. J. Phys. Chem. C 114 (2010) 12370 12374.

Soulié, J.-P.; Renaudin, G.; Černý, R.; Yvon, K.: Lithium boro-hydride $\mathrm{LiBH}_{4}$. I. Crystal structure. J. Alloys Compd. 346 (2002) 200205.

Spek, A. L. PLATON; University of Utrecht: Utrecht, The Netherlands, 2006.

Stinton, G. W.; Evans, J. S. O.: Parametric Rietveld refinement. J. Appl. Cryst. 40 (2007) 87-95.

Suryanarayana, C.: Mechanical alloying and milling. Progress in Mat. Sci. 46 (2001) 1-184.

Verdal, N; Hartman, M. R.; Jenkins, T.; DeVries, D. J.; Rush, J. J.; Udovic, T. J.: J. Phys. Chem. C 114 (2010) 10027-10033.

Vogel, S.; Ehm, L.; Knorr, K.; Braun, G.: Automated processing of 2D powder data. Adv. X-ray Anal. 45 (2002) 31-33.

Whitfield, P. S.: Spherical harmonics preferential orientation corrections and structure solution from powder diffraction data - a possible avenue of last resort. J. Appl. Cryst. 42 (2009) 134-136.

Woodley, S. M.; Battle. P. D.; Gale J. D.; Catlow, C. R. A.: The prediction of inorganic crystal structures using a genetic algorithm and energy minimization. Phys. Chem. Chem. Phys. 1 (1999) $2535-2542$. 